\title{
Efectividad y seguridad del esquema genérico lamivudina/tenofovir y efavirenz en pacientes con VIH/SIDA naïve: estudio fase IV no aleatorizado, Cali-Colombia 2012-2014
}

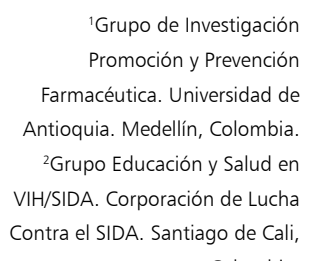

Colombia.

Los autores declaran no tener conflicto de intereses alguno en relación con el artículo publicado.

Disponibilidad de datos e información adicional. La totalidad de datos que soportan las conclusiones de este artículo están disponibles por solicitud al investigador principal (Dr. Jaime Galindo), Corporación de Lucha

Contra el Sida, Carrera 56

№ 2-120, Cali, Colombia;

Correo electrónico:

jaimegalindo@cls.org.co centroinvestigaciones@cls.org.co

El Grupo de Investigación Promoción y Prevención Farmacéutica recibió apoyo

financiero del Comité para el Desarrollo de Investigación (CODI)

y el Programa de Sostenibilidad

(2018-2019), Universidad de Antioquia, Medellín, Colombia.

Por su parte, el Grupo de Investigación "Educación y Salud

en VIH/Sida" recibió apoyo de la Corporación de Lucha contra el Sida.

Recibido: 13 de febrero de 2018 Aceptado: 26 de noviembre de 2018

Correspondencia a: Pedro Amariles pedro.amariles@udea.edu.co

Pedro Amariles", Jaime Galindo², Héctor F Mueses-Marín² y Carol Castañeda

Effectiveness and safety of generic version of lamivudine/tenofovir and efavirenz in treatment naïve HIV-infected patients: a nonrandomized, open-label, phase IV study in Cali-Colombia, 2012-2014

Background: Clinical studies aimed to evaluating the quality of generic drugs may be useful to strengthen policies of access to combined antiretroviral therapy (cART). Aim: To describe the effectiveness and safety of the generic schema lamivudine/tenofovir/efavirenz (3TC/TDF/EFV) in patients with HIV/AIDS naive, belonging to a comprehensive care program. Methods: A nonrandomized, open-label, phase IV study, during 2012 to 2014 naïve HIV-infected patients 18 years or older with indication to receive cART were recruited. Patients were treated with generic scheme 3TC/TDF/EFV and were followed-up during 12 months. Clinical, immunological and laboratory parameters were assessed at baseline, 3, 6 and 12 months of treatment. Results: Of the 40 patients, $30(75 \%)$ met the 12 months of treatment; of them, $80 \%$ achieved undetectable viral load ( $<40$ copies $/ \mathrm{mL})$ and $83.3 \%$ viral load $<50$ copies $/ \mathrm{mL}$. Additionally, there was a significant increase $\left(173 \mathrm{cells} / \mathrm{mm}^{3}\right)$ in the median for CD4 Tlymphocyte count. Moreover, the results of the whole blood count, creatinine and transaminases were preserved in normal ranges and did not generate changes in the cART. Potential side effects of antiretroviral drugs occurred in less than $10 \%$ of patients and had no serious implications. Conclusions: In this small group of patients, the generic scheme 3TC/ $\mathrm{TDF} / \mathrm{EFV}$ is effective and safe in the treatment of patients with HIV/AIDS naïve, and its effectiveness and safety profile is similar to show by innovator scheme $3 \mathrm{TC} / \mathrm{TDF} / \mathrm{EFV}$ in patients with similar clinical conditions. Registro Estudio: Registro Público Cubano de Ensayos Clínicos (RPCEC) ID: RPCEC00000134. Registered 20 July 2012.

Keywords: HIV/AIDS; generic drugs; antiretroviral drugs; phase IV study.

Palabras clave: VIH/SIDA; medicamentos genéricos; fármacos anti-retrovirales; estudios fase IV.

\section{Introducción}

L a infección por el virus de la inmunodeficiencia humana (VIH) y el síndrome de inmunodeficiencia adquirida (SIDA) continúan siendo una prioridad de salud pública. Globalmente, para el 2016 se estimó que 36,7 millones de personas estaban infectadas por VIH y un millón murieron por causas relacionadas al SIDA. Por su parte, para junio de 2017, 20,9 millones de personas que tenían infección por VIH tenían acceso a terapia antiretroviral combinada (TARc), indicando una cobertura de $57 \%$ de las personas infectadas con el virus ${ }^{1}$.

En Colombia, las metas nacionales buscan:

- 70\% de diagnósticos de la estimación de personas infectadas.

- $90 \%$ de cobertura con TARc en las personas con VIH.

- Una carga viral (CV) menor a 1.000 copias ARN/mL, en $90 \%$ de los pacientes con TARc ${ }^{2}$.
El logro de estos objetivos está determinado por la disponibilidad, acceso y adherencia al TARc ${ }^{3}$. Además, se estima que $64 \%$ de los costos de la atención a estos pacientes es generado por los medicamentos ${ }^{4}$ y que 20 a $40 \%$ de los pacientes que inician TARc no alcanzan las metas terapéuticas de supresión viral, entre otras causas, por el costo de los medicamentos ${ }^{3,5}$.

Tenofovir (TDF) ha evidenciado su efectividad en la prevención y tratamiento de la reactivación del virus de hepatitis $\mathrm{B}$ (VHB) en pacientes con infección por $\mathrm{VIH}^{6}$. En este contexto, en una cohorte de 100 pacientes con la co-infección VIH/VHB, el esquema lamivudina/ tenofovir/efavirenz (3TC/TDF/EFV) logró una supresión viral de 71 y $90 \%$ del VHB y del VIH, respectivamente ${ }^{7}$. Por ello, la combinación 3TC/TDF hace parte de las recomendaciones de la Organización Mundial de la Salud (OMS) para el tratamiento de la infección por VIH en pacientes con $\mathrm{VHB}^{8}$. 
La enfermedad por VIH/SIDA es de alto costo y requiere un tratamiento integral, incluyendo la disponibilidad y el acceso de medicamentos eficaces, seguros y económicos, situación en la que resulta clave una política farmacéutica orientada a favorecer la comercialización y distribución de medicamentos genéricos ${ }^{9}$. Sin embargo, esta política se asocia a cuestionamientos de calidad y a los resultados clínicos alcanzados en la práctica clínica. Adicionalmente, en Colombia, los estudios de bioequivalencia in vitro, basados en la comparación de los perfiles de disolución, entre medicamentos innovadores y genéricos ${ }^{10}$, son una alternativa aceptada, para sustentar la equivalencia terapéutica de medicamentos genéricos y lograr su autorización de comercialización ${ }^{11}$. Por ello, es necesario evaluar los resultados clínicos alcanzados con los medicamentos genéricos disponibles, realizando estudios diseñados a valorar los resultados de efectividad y seguridad alcanzados en los pacientes en los que se utilizan estos productos y, con ello, respaldar su utilización ${ }^{12}$. Por tanto, el objetivo de este trabajo fue describir la efectividad y seguridad del esquema genérico 3TC/EFV/TDF en pacientes con infección por $\mathrm{VIH} / \mathrm{SIDA}$ naïve, pertenecientes a un programa de atención integral especializado.

\section{Materiales y Métodos}

\section{Diseño}

Estudio prospectivo fase IV, tipo ensayo clínico, abierto, no controlado y de una sola rama. El grupo de estudio fue 40 pacientes con diagnóstico confirmado de infección por VIH, sin exposición previa a TARc, que requerían tratamiento y tenían contraindicación(es) para utilizar zidovudina (AZT) o abacavir $(\mathrm{ABC})$ como primera línea.

\section{Población y muestra}

Pacientes de ambos sexos, mayores de 18 años, con diagnóstico confirmado de infección por VIH/SIDA, sin exposición previa a TARc, pertenecientes al Programa de Atención Integral Especializada Ambulatoria de la Corporación de Lucha Contra el SIDA (CLS) de Santiago de Cali-Colombia.

El tamaño de muestra se estimó con las siguientes consideraciones: tasa de abandono y porcentaje de fracaso virológico de 20 y 15\%, respectivamente (acorde a estudios institucionales, previos con pacientes similares ${ }^{5,12}$; potencia de $80 \%$ y nivel de confianza de $95 \%$. Para ello, utilizando el software estadístico Epidat 4.0-módulo tamaño de muestra comparación de proporciones emparejadas, se determinó un mínimo de 33 pacientes para encontrar cinco fracasos por tratamiento ${ }^{12,13}$. Sin embargo, anticipando una tasa de retiro de $20 \%$, el tamaño de muestra estimado fue de 40 pacientes. Los pacientes que cumplieron los criterios de inclusión y firmaron el consentimiento informado, fueron reclutados y seleccio- nados por muestreo no probabilístico, entre julio de 2012 y febrero de 2013.

\section{Criterios de inclusión}

- Diagnóstico de infección por VIH: Por pruebas presuntivas y test confirmatorio (recuento de linfocitos $\mathrm{T}$ $\mathrm{CD} 4$, en céls $/ \mathrm{mm}^{3}$, por citometría de flujo, y valores de carga viral, en copias de ARN viral $/ \mathrm{mL}$, por ensayo de reacción de polimerasa en cadena en tiempo real (RPC-TR) de la transcriptasa reversa).

- Mayores de 18 años, de ambos sexos, sin exposición previa a TARc, que aceptaron participar voluntariamente mediante consentimiento informado.

- Con indicación y disposición para iniciar TARc.

- Con resultado genotípico negativo de resistencias a los fármacos del TARc.

\section{Criterios de exclusión}

- Fallo renal: Aclaramiento estimado de creatinina menor a $60 \mathrm{~mL} /$ minuto y/o creatininemia superior a $1,5 \mathrm{mg} / \mathrm{dL}$.

- Fallo hepático primario o asociado.

- Mujeres en gestación, lactancia o en edad reproductiva sin método anticonceptivo seguro o definitivo.

- Uso de medicamentos con probabilidad alta de interacciones de relevancia clínica.

- Pacientes hospitalizados.

- Presencia de trastornos psiquiátricos mayores.

- Antecedentes y/o problemas no intervenidos de drogadicción.

- Horarios de trabajo variables con turnos nocturnos.

\section{Criterios de descontinuación o retiro del estudio}

- Pérdida de seguimiento por muerte, abandono del estudio.

- Problemas graves relacionados al uso de los medicamentos y eventos adversos que requieran modificación o interrupción del tratamiento.

- Incumplimiento del tratamiento superior a $10 \%$ de las dosis prescritas.

Los pacientes fueron asignados al esquema genérico 3TC $150 \mathrm{mg}$ ( 2 comprimidos al día $=1$ cada $12 \mathrm{~h})+$ TDF $300 \mathrm{mg}$ (1 comprimido al día) + EFV $600 \mathrm{mg}$ (1 comprimido al día) (fabricados por Farmatech S.A para Humax Pharmaceutical S.A., Medellín-Colombia) y fueron seguidos durante doce meses.

Los medicamentos necesarios para el tratamiento de los pacientes fueron adquiridos por la Corporación de Lucha Contra el SIDA CLS de Santiago de CaliColombia, directamente a Humax Pharmaceutical S.A. Estos medicamentos, acorde con la normativa colombiana, contaban con su respectivo registro sanitario, los que fueron expedidos, con posterioridad a disponer de la evidencia de equivalencia terapéutica con los respec- 
tivos medicamentos innovadores, mediante estudios de bioequivalencia in vitro ${ }^{11}$, basada en la comparación de los perfiles de disolución ${ }^{10}$.

\section{Evaluación de efectividad y seguridad}

Para evaluar la efectividad se utilizaron valores de CV del ARN del VIH-1 (copias de ARN/mL) y el recuento de linfocitos T CD4 (céls $/ \mathrm{mm}^{3}$ ) cuantificados al inicio del estudio y a los 3, 6 y 12 meses. En detalle, la efectividad se definió como una $\mathrm{CV}<40$ copias $\mathrm{ARN} / \mathrm{mL}$, evaluada a los 12 meses de la línea de base. La CV se cuantificó por RPC-TR de la transcriptasa inversa. "Abbott Real Time HIV-1 m2000 assay (Abbott, Chicago, IL), con un límite de sensibilidad de 40 copias ARN/mL". Por su parte, el recuento de LT CD4 se determinó por citometría de flujo, (clitómetro de flujo FACS CANTO II). Para ello, se evaluó la linealidad del sistema BD FACS CANTO para concentraciones de leucocitos entre 0 y $3,3 \times 10^{4}$ de leucocitos $/ \mathrm{mm}^{3}$, y se evidenció linealidad para resultados entre 1 a 3.669 céls $/ \mathrm{mm}^{3}$. Las muestras de sangre fueron tomadas por el laboratorio clínico de la Cruz Roja, seccional Valle-Colombia, en la CLS, siguiendo el mismo procedimiento y estándares.

Para evaluar la seguridad se utilizaron:

- Pruebas de función hepática (transaminasas -aspartato aminotransferasa y alanino aminotransferasa-).

- Función renal (creatininemia y el aclaramiento de creatinina estimado, método de Cockcroft-Gault).

- Hemograma completo (recuento diferencial de eritrocitos y leucocitos, plaquetas, hemoglobina y hematocrito).

- Perfil lipídico completo (colesterol total, colesterol de baja densidad -LDLc-, colesterol de alta densidad -HDLc-y triglicéridos).

- Glucosa, fostatos, calcio.

- Circunferencias de cintura y cadera.

Excepto para el perfil lipídico, glucosa, fosfato y calcio, valorados basalmente y a los meses 6 y 12, las demás se cuantificaron en la línea base y a los 3, 6, y 12 meses. Adicionalmente, en cada consulta médica y cita de seguimiento fármaco-terapéutico, los pacientes fueron indagados por la presencia de los eventos adversos más comunes (erupción cutánea, mareos y pesadillas) del TARc. Los eventos adversos se clasificaron como graves (cambio o suspensión de tratamiento), moderados (necesidad de tratamiento farmacológico) o leves (información y educación para minimizar presentación) ${ }^{14}$. Por su parte, el médico y el farmacéutico, establecieron la causalidad de los eventos adversos, en especial los valorados como graves, utilizando el algoritmo de Naranjo y cols ${ }^{15}$.

\section{Seguimiento y recolección de datos}

Los datos fueron recolectados, a través de seguimiento clínico durante los 12 meses de tratamiento con el esquema $3 \mathrm{TC} / \mathrm{TDF} / \mathrm{EFV}^{14}$, y registrados en la historia clínica y en los formatos de recolección, al inicio y a los 3, 6 y 12 meses. En cada examen médico se tomaron muestras de sangre para valorar la seguridad. El seguimiento físico y clínico lo realizó un médico entrenado y con experiencia en el tratamiento de pacientes con infección por VIH/ SIDA. Por su parte, el seguimiento fármaco-terapéutico fue realizado por un farmacéutico entrenado en esta práctica, utilizando el método Dáder ${ }^{16}$. De forma global, el método Dáder es un proceso sistemático, orientado a resolver problemas de proceso o de resultado relacionados con los medicamentos en paciente con infección por VIH/SIDA ${ }^{17}$.

La disposición para iniciar el tratamiento y la escala de depresión CES-D se valoraron utilizando cuestionarios validados para ello ${ }^{18-20}$. La adherencia fue valorada con el cuestionario simplificado de adherencia a la medicación $(\mathrm{SMAQ})^{21}$.

\section{Análisis estadístico}

Las medidas de tendencia central, dispersión, tablas de frecuencia y gráficos Box-Plot fueron calculadas por un análisis exploratorio; además, se utilizó la prueba de Kolmogorov-Smirnof para establecer la normalidad de las variables. Los datos iniciales fueron comparados con los valorados en los tiempos 3, 6 y 12 meses, utilizando el test de rango con signo de Wilcoxon para proporciones y porcentajes, o la prueba de t de Student o ANOVA para mediciones repetidas de variables continuas. Valores de $p<0,05$ fueron considerados estadísticamente significativos. Los análisis se realizaron utilizando el programa STATA versión 12.1. College Station, Texas USA.

\section{Consideraciones éticas}

Este estudio se ajustó a las normas mundiales de ética vigentes, Declaración de Helsinki y sus enmiendas, y la Guía de las Buenas Prácticas Clínicas (ICH E6). Respecto a la normatividad colombiana, se acogió a lo dispuesto en la Resolución 8430/93 y la Resolución 2378/2008. El estudio fue aprobado por el Comité de Ética de la CLS (Comité de Ética para Investigación en Humanos de la Corporación de Lucha Contra el SIDA-IRB 00005732at U.S. Department of Health and Human Services) y la participación de los pacientes fue voluntaria y manifiesta a través del consentimiento informado.

\section{Resultados}

\section{Características socio-demográficas}

El flujo de pacientes durante el estudio se describe en la Figura 1. En total, 47 pacientes fueron invitados a participar, de ellos 40 fueron incluidos y 30 completaron los 12 
meses de seguimiento. Los 40 pacientes incluidos fueron de recursos económicos bajos, en su mayoría $(92,5 \%)$ afiliados al sistema de salud subsidiado; además presentaban características de habitantes de calle o violencia social. De ellos $10(25 \%)$ presentaron razones para retiro del estudio (Figura 1). Las características demográficas y clínicas de línea base de los 30 pacientes se presentan en la Tabla 1. La media de edad fue de 33,8 \pm 8,5 años y $86,7 \%$ fueron hombres. De estos 30 pacientes al inicio del estudio cuatro (13\%) tenían resultado positivo del antígeno de superficie para hepatitis $\mathrm{B}\left(\mathrm{Ag}_{\mathrm{S}} \mathrm{HB}\right)$. De estos cuatro pacientes, en uno de ellos dicho marcador fue negativo al final del estudio.

\section{Efectividad del esquema genérico 3TC/TDF/EFV}

A los 12 meses, 24 de 40 (60,0\%), y 24 de 30 (80,0\%) pacientes, en análisis de intención a tratar y por protocolo, respectivamente, lograron una $\mathrm{CV}$ de $\mathrm{VIH}-1<40$ copias ARN/mL (Tabla 2, Figura 2A). Por su parte, entre la línea base y los 12 meses, hubo un incremento en la mediana de $173 \mathrm{LC} \mathrm{CD} 4 / \mathrm{mm}^{3}$ (Tabla 2); además, el recuento de LT CD4/ $/ \mathrm{mm}^{3}$ aumentó en promedio 207,5 $\pm 129,6$ céls/ $\mathrm{mm}^{3}, \mathrm{p}<0,001$ (datos no mostrados). Igualmente, entre el inicio y 12 meses, se observó un incremento de 11,0\% en la mediana del porcentaje de LT CD4 [19,7 (10,2-27,9) vs $30,5(25,6-42,0), \mathrm{p}<0,001]$ (Tabla 2, Figura 2B). Al final del seguimiento, $53 \%$ de los pacientes presentaron

\section{Pacientes sin exposición previa a TARc "naïve" $(n=47)$}

No cumplen criterios de inclusión $(n=7)$

- Un paciente por antecedente de exposición a tratamiento anti-retroviral (información no identificada en el tamizaje previo).

- Dos pacientes con perfil de disposición inadecuada para inicio de TARc y bajo perfil de adherencia.

- Tres pacientes por presencia en el estudio genotípico de resistencias de al menos una mutación que confiere resistencia potencial a uno de los medicamentos del protocolo.

- Un paciente por decisión voluntaria de no iniciar TARc.

Pacientes que iniciaron esquema de estudio $(\mathbf{n}=40)$
- Cuatro por incumplimiento del tratamiento por parte del paciente.
- Uno por intolerancia al efavirenz.

- Uno por intolerancia al efavirenz.

Pacientes que continuaron después del $3^{\text {er }}$ mes $(\mathbf{n}=35)$
Abandonos entre 3 y 6 meses $(\mathbf{n}=1)$
- Uno por incumplimiento del tratamiento por parte del paciente.

Pacientes que continuaron después del $6^{\circ}$ mes $(n=34)$
Abandonos entre 6 y 12 meses $(n=4)$
- Dos por incumplimiento del tratamiento por parte del paciente.
- Uno por muerte violenta.
- Uno no asistió a toma de exámenes de seguimiento.

- Uno no asistió a toma de exámenes de seguimiento.

\section{Pacientes que finalizaron el tratamiento $(n=30)$}

Figura 1. Tamizaje de pacientes bajo estudio. TARc: tratamiento anti-retroviral combinado

\begin{tabular}{|c|c|c|c|}
\hline Características de los pacientes & & $n=40$ & $\mathrm{n}=30$ \\
\hline Edad, media (desviación estándar), en años & $\begin{array}{l}\text { Total } \\
\text { Hombre } \\
\text { Mujer }\end{array}$ & $\begin{array}{lr}34,1 & (9,9) \\
33,8 & (9,5) \\
36,0 & (13,2)\end{array}$ & $\begin{array}{ll}33,8 & (8,5) \\
34,3 & (8,9) \\
30,2 & (3,7)\end{array}$ \\
\hline Género, n (\%) & $\begin{array}{l}\text { Hombre } \\
\text { Mujer }\end{array}$ & $\begin{array}{rr}35 & (87,5) \\
5 & (12,5)\end{array}$ & $\begin{array}{rr}26 & (86,7) \\
4 & (13,3)\end{array}$ \\
\hline Raza, n (\%) & $\begin{array}{l}\text { Afroamericano } \\
\text { Blanco }\end{array}$ & $\begin{array}{rr}4 & (10,0) \\
36 & (90,0)\end{array}$ & $\begin{array}{rr}3 & (10,0) \\
27 & (90,0)\end{array}$ \\
\hline Estado civil, n (\%) & $\begin{array}{l}\text { Casado-unión libre } \\
\text { Soltero-separado }\end{array}$ & $\begin{array}{rr}8 & (20,0) \\
32 & (80,0)\end{array}$ & $\begin{array}{rr}8 & (26,6) \\
22 & (73,3)\end{array}$ \\
\hline Educación, n (\%) & $\begin{array}{l}\text { Primaria } \\
\text { Secundaria } \\
\text { Educación técnica } \\
\text { Universitaria }\end{array}$ & $\begin{array}{rr}13 & (32,5) \\
19 & (47,5) \\
2 & (5,0) \\
6 & (15,0)\end{array}$ & $\begin{array}{rr}10 & (33,3) \\
13 & (43,3) \\
2 & (6,7) \\
5 & (16,7)\end{array}$ \\
\hline Ocupación, n (\%) & $\begin{array}{l}\text { Hogar } \\
\text { Estudiante } \\
\text { Desempleado } \\
\text { Empleado }\end{array}$ & $\begin{array}{rr}3 & (7,5) \\
3 & (7,5) \\
12 & (30,0) \\
22 & (55,0)\end{array}$ & $\begin{array}{rr}3 & (10,0) \\
2 & (6,7) \\
9 & (30,0) \\
16 & (53,3)\end{array}$ \\
\hline Centros para el Control y Prevención de Enfermedades - etapas clínicas & $\begin{array}{l}\text { A } \\
B \\
C\end{array}$ & $\begin{array}{ll}18 & (45,0) \\
12 & (30,0) \\
10 & (25,0)\end{array}$ & $\begin{array}{rr}13 & (43,3) \\
9 & (30,0) \\
8 & (26,7)\end{array}$ \\
\hline
\end{tabular}


Tabla 2. Variables clínicas relacionadas con la evaluación de la efectividad (ingresaron n = 40, completaron el estudio n = 30), Cali-Colombia, 2012-2015

\begin{tabular}{|c|c|c|c|c|c|}
\hline Variables clínicas & Línea & ase & 3 meses & 6 meses & 12 meses \\
\hline Carga viral copias ARN/mL & $n=40$ & $\mathrm{n}=30$ & $\mathrm{n}=30$ & $\mathrm{n}=30$ & $\mathrm{n}=30$ \\
\hline Mediana (rango intercuartil, Q1-Q3) & $60.860,5(14.770,5-179.474,5)$ & $50.588,0(14.261-168.962)$ & $0(0-137)$ & $0(0-47)$ & $0(0-0)$ \\
\hline$\leq 40$ copias, $\mathrm{n}(\%)^{\mathrm{a}}$ & $0(0,0)$ & $\begin{array}{lll}0 & (0,0)\end{array}$ & $20(57,1)$ & $23(67,7)$ & $24(80,0)$ \\
\hline$\leq 50$ copias, $\mathrm{n}(\%)^{\mathrm{a}}$ & $0 \quad(0,0)$ & $0 \quad(0,0)$ & $20(57,1)$ & $24(80,0)$ & $25(83,0)$ \\
\hline 41-400 copias, n (\%) & $0 \quad(0,0)$ & $0 \quad(0,0)$ & $13(37,1)$ & $11(32,3)$ & $6(20,0)$ \\
\hline $401-1.000$ copias, $n(\%)$ & $0 \quad(0,0)$ & $0 \quad(0,0)$ & $2(5,7)$ & $0(0,0)$ & $0 \quad(0,0)$ \\
\hline $1.001-10.000$ copias, $\mathrm{n}(\%)$ & $7(17,5)$ & $5(16,7)$ & $0 \quad(0,0)$ & $0 \quad(0,0)$ & $0 \quad(0,0)$ \\
\hline $10.001-1.000 .000$ copias, $\mathrm{n}(\%)$ & $33(82,5)$ & $25(83,3)$ & $0(0,0)$ & $0 \quad(0,0)$ & $0 \quad(0,0)$ \\
\hline
\end{tabular}

Recuento de linfocitos T CD4 (céls $/ \mathrm{mm}^{3}$ )

\begin{tabular}{|c|c|c|c|c|c|}
\hline$\geq 200$ n (\%) & $25(62,5)$ & $19(63,3)$ & $25(83,3)$ & $27(90,0)$ & $28(93,3)$ \\
\hline Mediana (rango intercuartil, Q1-Q3)a & $270,5(107-412)$ & $288,5(106-410)$ & $364,5(261-511)$ & $364,0(270-631)$ & $443,5(305-682)$ \\
\hline Mínimo & 7 & 7 & 39 & 67 & 100 \\
\hline Máximo & 1.146 & 825 & 981 & 1.089 & 1.091 \\
\hline \multicolumn{6}{|l|}{ nfocitos T CD4 (\%) } \\
\hline Mediana (rango intercuartil, Q1-Q3) ${ }^{\mathrm{a}}$ & $19,7(10,2-27,9)$ & $19,7(10,7-25,9)$ & $24,8(19,1-34,0)$ & $28,5(23,0-33,0)$ & $30,5(25,6-42,0)$ \\
\hline Mínimo & 1,5 & 1,5 & 6,7 & 12,1 & 17,0 \\
\hline Máximo & 42,9 & 36,0 & 44,0 & 52,0 & 80,0 \\
\hline
\end{tabular}

${ }^{a}$ valor-p $<0,0011$, para diferencias entre línea base, 3, 6, y 12 meses.
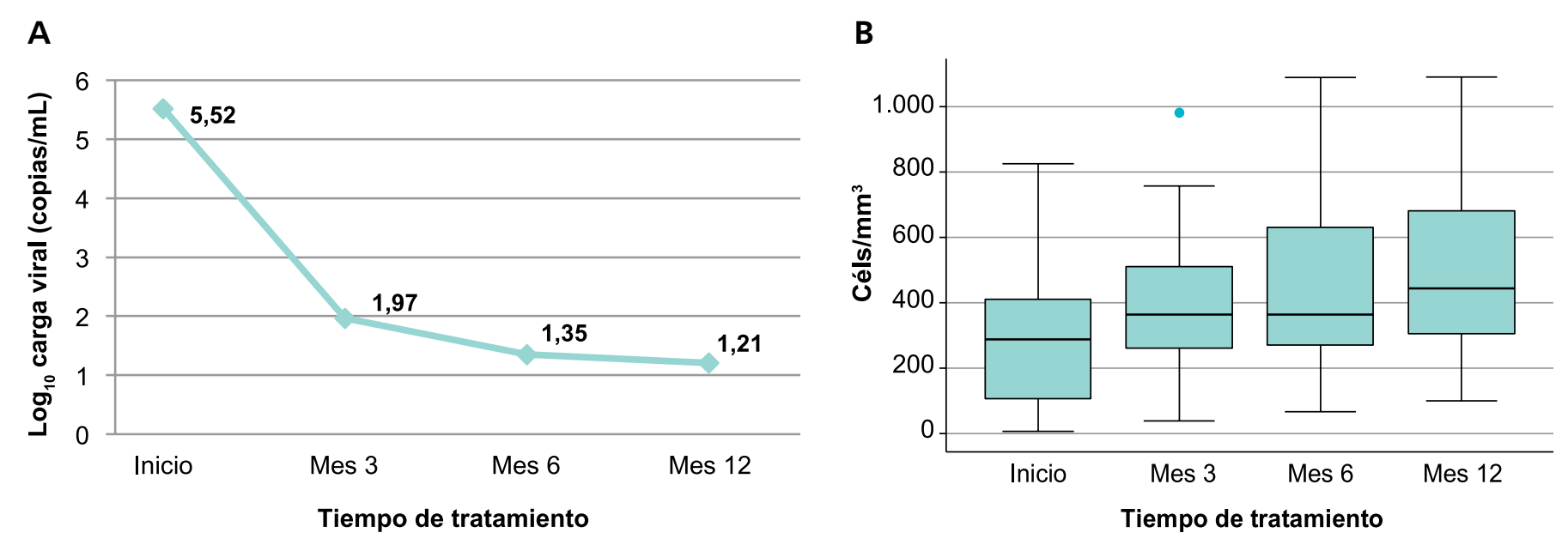

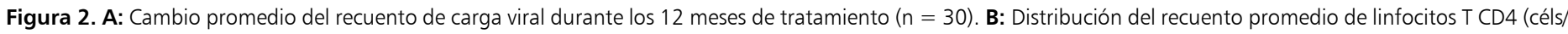
$\left.\mathrm{mm}^{3}\right)$ durante 12 meses de tratamiento $(n=30)$.

$\mathrm{CV}<40$ copias ARN/mL y LT CD4 > 350 céls $/ \mathrm{mm}^{3}$ (datos no mostrados).

\section{Seguridad del esquema genérico TDF/3TC/EFV}

De los 40 pacientes del estudio, 10 (25\%) presentaron razones para retiro del estudio: Un paciente presentó intolerancia al EFV y otro falleció en una muerte violenta (homicidio) durante el seguimiento. La exclusión de los otros ocho pacientes se debió a: siete por abandono del tratamiento no asociado a intolerancia o efectos adversos de la TARc, y uno por falta de adherencia al seguimiento y controles (Figura 1). Por tanto, de los 10 retiros del estudio, uno (10\%) fue por causas atribuidas al TARc, intolerancia grave al efavirenz (depresión y pesadillas notorias). En este caso, la causalidad de la reacción adversa fue probable (5 puntos, algoritmo de Naranjo y cols) ${ }^{15}$. Por su parte, los efectos indeseables más frecuentes o eventos adversos, pero sin interrupción del tratamiento 
(moderados o leves), fueron: erupción cutánea, síndrome metabólico, pesadillas y mareo en cuatro $(13 \%)$, tres $(10 \%)$, dos $(7 \%)$ y en un paciente $(3 \%)$, respectivamente. Durante los 12 meses de seguimiento no hubo cambios relevantes en la función hepática, renal o hematológica en los pacientes (Tabla 3). En este contexto, los resultados muestran ausencia de variaciones significativas entre las mediciones iniciales y finales de las transaminasas hepáticas. Los valores de hemoglobina incrementaron significativamente, manteniéndose dentro de los rangos aceptables en todos los pacientes. Los valores de creatininemia mostraron una disminución significativa $(p=0,0013)$.

\section{Lípidos y otros parámetros de laboratorio}

En los pacientes hubo un aumento significativo, pero en rangos de normalidad, en colesterol total, LDLc y glicemia; además, se observó aumento significativo en las concentraciones de HDLc (Tabla 3). Por su parte, las concentraciones de triglicéridos, fosfatos, calcio y mediciones de cintura no presentaron cambios significativos (datos no mostrados).

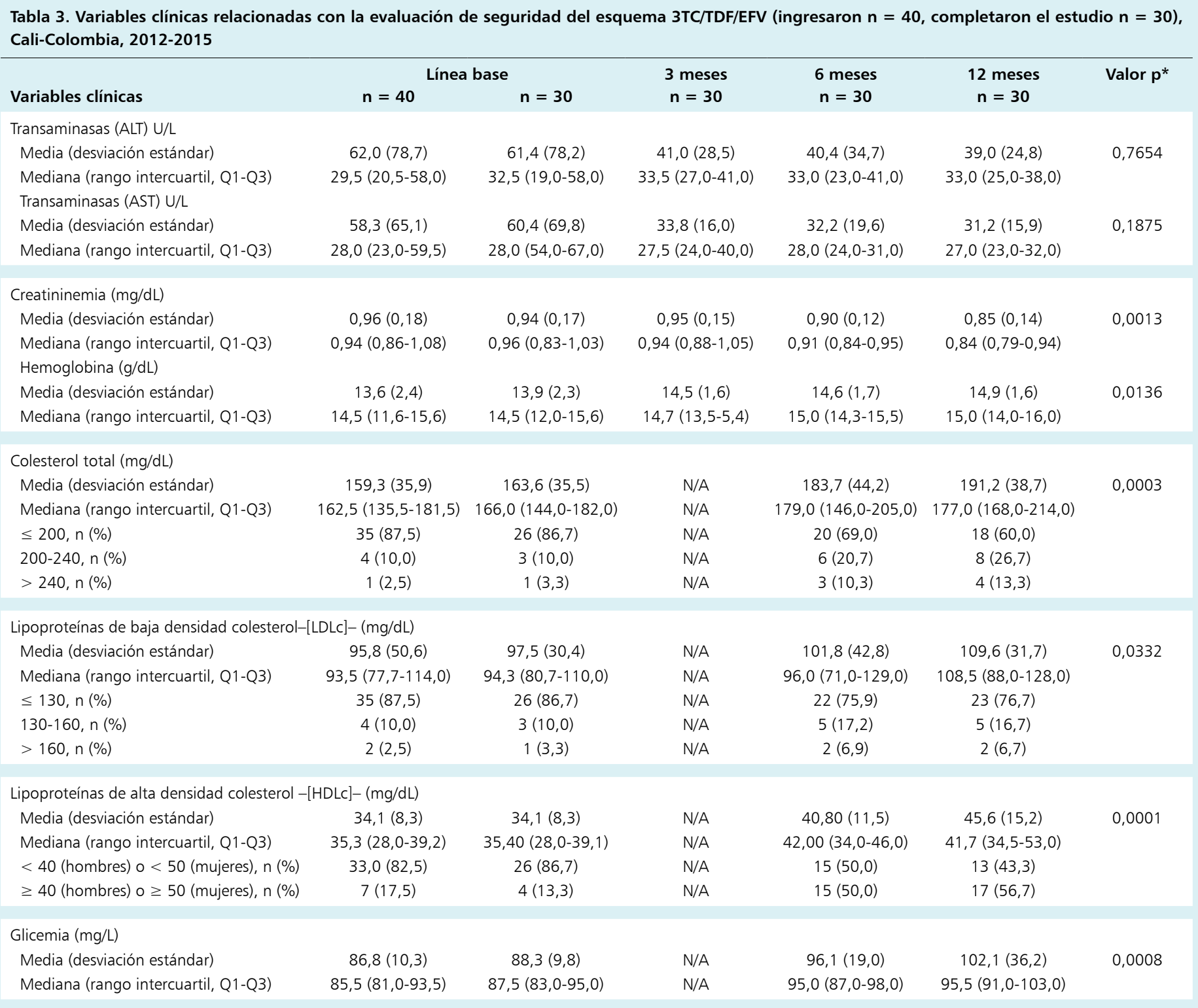

*Para diferencias entre línea base y 12 meses. N/A: no evaluado; ALT: alanina aminotransferasa; AST aspartato aminotransferasa. 


\section{Discusión}

Los 40 pacientes incluidos en este estudio se caracterizaron por condiciones socio-económicas muy adversas, incluyendo condiciones económicas precarias, habitantes de calle, informalidad laboral, abuso de sustancias y violencia social; situación que podría explicar la tasa alta de abandonos $(25 \%)^{22-24}$. En este sentido, 9 de los 10 abandonos, incluyendo uno por homicidio, podrían deberse a esta situación; mientras que sólo uno (10\%) se asoció al TARc, específicamente intolerancia a efavirenz (Figura 1).

Relacionado con la efectividad, en el análisis por protocolo (30 pacientes que completaron los 12 meses), el esquema genérico 3TC/TDF/EFV generó una reducción significativa de la CV. En este sentido, de los 30 pacientes, 80 y $83,3 \%$ alcanzaron valores de $\mathrm{CV}$ menores a las 40 y 50 copias ARN/mL, respectivamente. Por su parte, si en el análisis de intención por tratar, se incluyera solamente los fallos (un paciente retirado por problemas de seguridad), la efectividad del TARc sería de 77,4\% (24/31). Adicionalmente, en el grupo de 30 pacientes hubo un incremento significativo de 173 céls $/ \mathrm{mm}^{3}$ en la mediana del recuento de LT CD4 (443,5 versus 270,5 céls $\left./ \mathrm{mm}^{3}\right)$.

Acorde con las recomendaciones del GeSIDA-Grupo de estudio del Sid ${ }^{25}$, la respuesta virológica al TARc se evidencia con una reducción en la CV mayor a $1 \log$ de base 10, a las cuatro semanas de tratamiento, y por un incremento mínimo de 50-100 LT CD4, durante el primer año. Un tratamiento que es efectivo genera:

- Disminución de la CV mínimo en un logaritmo en base 10, a las 6-8 semanas.

- Incremento aproximado de $100-150 \mathrm{LT} \mathrm{CD} 4 / \mathrm{mm}^{3}$ por año.

- Retraso en la aparición de infecciones oportunistas ${ }^{17}$.

En este sentido, el esquema genérico 3TC/TDF/EFV fue efectivo, debido a que generó: supresión viral superior a los dos logaritmos al tercer mes de tratamiento (Figura $2 \mathrm{~A})$; supresión viral máxima $(\mathrm{CV}<40$ copias $\mathrm{ARN} /$ $\mathrm{mL})$ en $80 \%(\mathrm{n}=24 / 30)$ de los pacientes a los 12 meses (Tabla 2); aumento igual o superior a 100 céls $/ \mathrm{mm}^{3}$ en $76,7 \%(n=23 / 30)$ (datos no mostrados) y; d) ausencia de infecciones oportunistas durante los 12 meses.

Relacionado con la seguridad, el uso de TDF podría causar reducción en la densidad ósea y/o incremento de la creatininemia y proteinuria ${ }^{26}$. Sin embargo, la magnitud de estos efectos es modesta y no genera restricciones en su indicación ${ }^{27,28}$. Por el contrario, en el presente estudio, se evidenció una disminución significativa $(\mathrm{p}=0,0013)$ en las concentraciones de creatinina sérica; lo que podría deberse al efecto del esquema completo utilizado; sin embargo, la interpretación adecuada de esta información requiere de estudios diseñados para valorar este aspecto. Por su parte, lamivudina presenta efectos adversos mínimos y efavirenz puede causar alteraciones del sistema nervioso central (pesadillas, depresión, confusión mental y mareos), ginecomastia y síndrome de Steven Johnson $^{14,29,30}$. De forma global, en el presente estudio, de los 10 pacientes retirados, sólo uno (10\%) fue por problemas de seguridad, específicamente por alteraciones graves del sistema nervioso central atribuidas al EFV (relación causal probable).

Relacionado con otros efectos adversos de este esquema $^{14,30}$, se identificó erupción cutánea $(13 \%)$, síndrome metabólico (10\%), pesadillas (7\%) y mareo (3\%). Estos valores fueron, acorde con lo esperado, valorados como leves o moderados (excepto el caso de suspensión asociada a EFV) y se resolvieron durante las primeras 2-4 semanas de iniciado el TARc.

En una revisión realizada el 30 de junio de 2017, en PubMed/MedLine, para identificar estudios de eficacia del esquema 3TC/TDF/EFV, utilizando la siguiente estrategia de búsqueda: tenofovir[Title] AND lamivudine[Title] AND efavirenz[Title] AND efficacy[Title]) AND ("loattrfull text"[sb] AND "humans"[MeSH Terms] AND (English[lang] OR Spanish[lang]), se identificaron siete posibles artículos. De ellos, un estudio de cohortes multicéntrico fase IV (ESAR 10-01: 1.608 pacientes de 13 centros de ocho países europeos) y realizado con el objetivo de comparar los resultados del esquema innovador 3TC/TDF/EFV (427 pacientes) con el esquema tenofovir emtricitabina y efavirenz (1.181 pacientes), fue considerado adecuado para comparar los resultados. En este sentido, en el grupo tenofovir, lamivudina y efavirenz, al año de tratamiento, se logró $\mathrm{CV}$ indetectable $(<50$ copias $\mathrm{ARN} / \mathrm{mL}$ ) en 67,2 y $92,3 \%$ de los pacientes, en intención por tratar y por protocolo, respectivamente ${ }^{31}$.

Aunque las diferencias del estudio referencia ${ }^{31}$ con el presente fueron notorias, los resultados de efectividad fueron similares, en términos de $\mathrm{CV}$ indetectable $(<40$ copias ARN/mL), 60,0 y $80,0 \%$ de los pacientes, en intención por tratar y por protocolo, respectivamente. Estos valores son más próximos, si la efectividad se establece con el mismo valor del estudio referencia (CV $<50$ copias ARN/mL), en el que se alcanzan valores de 62,5 y $83,3 \%$ de los pacientes, en intención por tratar y por protocolo, respectivamente. Además, en el grupo de pacientes, se logró un aumento de la mediana inicial de 173 céls $/ \mathrm{mm}^{3}$ (Tabla 2). Por tanto, se podría establecer que los resultados de efectividad del esquema genérico 3TC/TDF/EFV del presente estudio son similares a los resultados reportados con el esquema innovador en el estudio referencia ${ }^{31}$ al año de tratamiento.

Las guías colombianas recomiendan, como opción preferida para iniciar TARc, el esquema TDF/FTC (o abacavir ABC/3TC) + EFV, debido a la co-formulación 
disponible de TDF/FTC y ABC/3TC ${ }^{14}$. Por su parte, la OMS en sus guías del 2013, recomienda la combinación TDF + 3TC (o TDF + emtricitabina-FTC) + EFV (idealmente a dosis fija), aclarando que los datos disponibles sugieren que 3TC y FTC pueden utilizarse indistintamen$t^{8}$. Aunque existen algunas diferencias farmacológicas, entre 3TC y FTC, en especial una vida media mayor de FTC (11 h versus las siete horas para 3TC) y, con ello, posibles ventajas de FTC; una revisión sistemática reciente concluyó que: los resultados farmacológicos de estudios clínicos, orientados a establecer comparaciones directas e indirectas, muestran que 3TC y FTC pueden considerarse terapéuticamente intercambiables y, además que, en el caso de existir diferencias, las mismas son mínimas y carecen de importancia clínica ${ }^{32}$. Por lo anterior, en pacientes con contraindicaciones para el uso de $\mathrm{ABC}$, la utilización del esquema 3TC/TDF/EFV puede ser una alternativa que, por su menor costo, podría favorecer una mayor cobertura en países con recursos limitados. En consecuencia, el presente estudio, diseñado con el objetivo de valorar la efectividad y seguridad del esquema genérico 3TC/TDF/EFV, tiene como valor adicional, la generación de resultados alcanzados con la utilización de una combinación menos común, al incluir 3TC y no FTC.

Las limitaciones de este estudio son importantes y, por tanto, los resultados y conclusiones deberían interpretarse con precaución antes de ser extrapolados a otros países. En este contexto, la tasa de abandonos por falta de adherencia al tratamiento redujo significativamente el tamaño muestral. Sin embargo, este aspecto se podría atribuir a causas ajenas al TARc, como fueron las condiciones adversas de extrema pobreza, inestabilidad laboral, violencia y abuso de sustancias. A pesar de esta limitación, en este grupo de pacientes, con condiciones socio-económicas tan adversas, en los que se logra adherencia al programa de atención integral, incluyendo el TARc, los resultados, en términos de efectividad y seguridad, son similares a los obtenidos en estudios previos con el mismo esquema de TARc con medicamentos innovadores. Además, este estudio, de forma similar a estudios previos ${ }^{12,13}$, provee información relevante de la efectividad y seguridad del esquema 3TC/ TDF/EFV genérico, lo que se asocia a una mayor confianza con la utilización de este tipo de productos. Por tanto, en este grupo pequeño de pacientes, el esquema genérico 3TC/TDF/EFV es efectivo y seguro en el tratamiento de la infección por VIH en pacientes naïve. Adicionalmente, la efectividad alcanzada con el esquema genérico durante un año es similar a reportada en la literatura médica para el mismo esquema con fármacos innovadores en pacientes con condiciones clínicas similares.

\section{Resumen}

Antecedentes: Los estudios clínicos orientados a evaluar la calidad de medicamentos genéricos pueden ser útiles para fortalecer políticas de acceso a terapia anti-retroviral combinada (TARc). Objetivo: Describir la efectividad y seguridad del esquema genérico lamivudina/ tenofovir/efavirenz (3TC/TDF/EFV) en pacientes con infección por VIH/SIDA naïve, pertenecientes a un programa de atención integral. Materiales/Métodos: Estudio clínico prospectivo fase IV abierto y sin grupo control. Entre 2012-2014, se incluyeron y siguieron 40 pacientes con infección por VIH/SIDA naïve y con indicación para iniciar tratamiento. Los pacientes fueron tratados con el esquema genérico 3TC/TDF/EFV y fueron seguidos durante 12 meses. El seguimiento incluyó valoración clínica, parámetros inmunovirológicos y de laboratorio, al inicio del tratamiento y a los 3, 6 y 12 meses. Resultados: De los 40 pacientes, $30(75 \%)$ cumplieron los doce meses de tratamiento; de ellos, $80 \%$ alcanzó CV indetectable $(<40$ copias $/ \mathrm{mL}$ ) y $83,3 \% \mathrm{CV}<50$ copias $/ \mathrm{mL}$. Adicionalmente, en el grupo hubo un incremento en la mediana de 173 linfocitos TCD4/ $\mathrm{mm}^{3}$. Por su parte, los resultados del hemograma completo, creatininemia y transaminasas hepáticas se conservaron en rangos normales y no generaron cambios del TARc. Los efectos adversos reconocidos para estos medicamentos se presentaron en menos de $10 \%$ de los pacientes y no tuvieron implicaciones graves. Conclusiones: En este grupo pequeño de pacientes, el esquema genérico 3TC/TDF/EFV es efectivo y seguro en el tratamiento de pacientes con infección por VIH/SIDA naïve, y su perfil de efectividad y seguridad es similar al del esquema 3TC/TDF/EFV innovador en pacientes con condiciones clínicas similares.

\section{Referencias bibliográfícas}

1.- ONUSIDA. Hoja informativa - Últimas estadísticas sobre el estado de la epidemia de sida. Ginebra, 2017. [Online] http://www. unaids.org/es/resources/fact-sheet. (Consultado el 16 de julio de 2018).

2.- Cuenta de Alto Costo. Fondo Colombiano de Enfermedades de Alto Costo. Situación del VIH-Sida en Colombia, 2015. Bogotá,
Colombia. [Online] https://cuentadealtocosto. org/site/images/Publicaciones/

Situacio\%CC\%81n\%20del\%20VIH\%20en\%20 Colombia\%202015.pdf (Consultado el 7 de julio de 2017).

3.- Machado J E, Alzate J A. Patrones de prescripción de antirretrovirales en 997 pacientes colombianos. Biomédica 2008; 28 : 78-86. DOI: https://doi.org/10.7705/biomedica. v28i1.110
4.- González-Mayorga M A. Evaluación del acceso a la terapia antirretroviral de las personas viviendo con VIH en el distrito capital desde una mirada crítica al sistema de salud colombiano. Tesis de Maestría en Salud Pública 2011. Grupo de Investigación: Antropologia Médica Crítica y Universidad Nacional de Colombia, sede Bogotá.

5.- Galindo-Orrego P, Mueses-Marín H F, Galindo-Quintero J, Martínez-Cajas J L. 
Resistencia transmitida del virus de la inmunodeficiencia humana en pacientes sin exposición previa a tratamiento antirretroviral, Cali, Colombia, 2014. Infectio 2013; 17 (1): 19-27. DOI: 10.1016/S01239392(13)70044-4.

6.- Koskinas J S, Deutsch M, Adamidi S, Skondra M, Tampaki M, Alexopoulou A, et al. The role of tenofovir in preventing and treating hepatitis $\mathrm{B}$ virus (HBV) reactivation in immunosuppressed patients. A real life experience from a tertiary center. Eur J Intern Med 2014; 25 (8): 768-71. DOI: 10.1016/j. ejim.2014.06.028.

7.- $\quad$ Wu Y S, Zhang W W, Ling X M, Yang L, Huang S B, Wang X C, et al. Efficacy and safety of tenofovir and lamivudine in combination with efavirenz in patients co-infected with human immunodeficiency virus and hepatitis B virus in China. Chin Med J (Engl) 2016; 129 (3): 304-8. DOI: 10.4103/0366-6999.174509.

8.- World Health Organization. Consolidated guidelines on the use of antiretroviral drugs for treating and preventing HIV infection. 2013 [Citado el 20 de abril de 2017]. Disponible en: http://www.who.int/hiv/pub/guidelines/ arv2013/en.

9.- Consejo Nacional de Política Económica y Social. Política Farmacéutica Nacional 2012, 2012 Bogotá, Colombia [Online] [https:// www.minsalud.gov.co/Documentos $\% 20$ y\%20Publicaciones/Politica $\% 20$ Farmac\%C3\%A9utica\%20Nacional.pdf. (Consultado el 7 de julio de 2017).

10.- Al Ameri M N, Nayuni N, Anil Kumar K G, Perrett D, Tucker A, Johnston A. The differences between the branded and generic medicines using solid dosage forms: in-vitro dissolution testing. Results Pharma Sci 2011; 2: 1-8. DOI: 10.1016/j. rinphs.2011.12.001.

11.- Ministerio de Salud de Colombia. Resolución 1400. 2001 [Citado el 20 de abril de 2017]. Disponible en: https://www.invima.gov. co/images/pdf/medicamentos/resoluciones/ resolucion-1400.pdf.

12.- Gutiérrez F J, Amariles P, Galindo J, Mueses H, Agudelo J A, Hincapié JA. Efectividad y seguridad del esquema genérico lamivudina/ zidovudina/efavirenz en pacientes $\mathrm{VIH}(+)$. Estudio fase IV y comparación con el mismo esquema de medicamentos innovadores. Vitae 2013; 20 (1): 30-40. http://www.scielo.org.co/ pdf/vitae/v20n1/v20n1a4.pdf.

13.- Galindo J, Amariles P, Mueses-Marín H F, Hincapié J A, González-Avendaño S, GalindoOrrego X. Effectiveness and safety of generic version of abacavir/lamivudine and efavirenz in treatment naive HIV-infected patients: a nonrandomized, open-label, phase IV study in Cali-Colombia, 2011-2012. BMC Infect Dis 2016; 16 (1): 532. DOI: 10.1186/s12879-0161871-x.

14.- Ministerio de Salud y Protección Social de Colombia. Guía de práctica clínica basada en la evidencia científica para la atención de la infección por VIH/Sida en adolescentes (con 13 años de edad o más) y adultos; 2014 [Citado el 20 de abril de 2017]. Disponible en: http:// gpc.minsalud.gov.co/gpc_sites/Repositorio/ Otros_conv/GPC_VIH_adolescentes/gpc_vih_ adolescentes_completa.aspx.

15.- Naranjo C A, Busto U, Sellers E M, Sandor P, Ruiz I, Roberts E A, et al. A method for estimating the probability of adverse drug reactions. Clin Pharmacol Ther 1981; 30: 239 45. PMID: 7249508.

16.- Pharmaceutical Care Research Group, University of Granada (Spain). Pharmacotherapy follow-up: the dader method (third revision: 2005). Pharm Pract 2006; 4 : 44-53.

17.- Amariles P. Seguimiento farmacoterapéutico en pacientes VIH/SIDA. Aula de Farmacia 2011; 7 (87): 8-24.

18.- Herrero J, Gracia E. Una medida breve de la sintomatología depresiva (CESD-7). Salud Mental 2007; 30: 40-6. http:/www. medigraphic.com/pdfs/salmen/sam-2007/ sam075g.pdf.

19.- Irwin M, Artin K H, Oxman M N. Screening for depression in the older adult: criterion validity of the 10-item Center for Epidemiological Studies Depression Scale (CES-D). Arch Intern Med 1999; 159 (15): 1701-4. PMID: 10448771.

20.- Radloff L S. The CES-D Scale: A SelfReport Depression Scale for research in the general population. Appl Psychol Meas 1977; 1 (3): 385-401. https://doi.org/10.1177\% 2F014662167700100306.

21.- Rodríguez Chamorro M A, García-Jiménez E, Amariles P, Rodríguez Chamorro A, Faus M J. Revisión de tests de medición del cumplimiento terapéutico utilizados en la práctica clínica. Aten Primaria 2008; 40 (8): 413-8. http://www. elsevier.es/es-revista-atencion-primaria-27pdf-13125407.

22.- Accorsi S, Fabiani M, Nattabi B, Corrado B, Iriso R, Ayella E O, et al. The disease profile of poverty: Morbidity and mortality in northern Uganda in the context of war, population displacement and HIV/AIDS. Trans R Soc Trop Med Hyg 2005; 99 (3): 226-33. DOI: 10.1016/j. trstmh.2004.09.008.

23.- Mabala R. From HIV prevention to HIV protection: addressing the vulnerability of girls and young women in urban areas. Environment
\& Urbanization 2006; 18 (2): 407-32. DOI: $10.1177 / 0956247806069624$.

24.- Malagón Rojas J N. VIH/SIDA en Colombia: Análisis de la relación entre la desigualdad social, salud y conflicto armado 2002-2008. (Sin mes) 2011. Investigación \& Desarrollo. 2011,19 (Consultado el 12 de julio de 2017) Online http://www.redalyc.org/articulo. oa?id=26820753001

25.- Panel de expertos de GeSIDA y Plan Nacional sobre el Sida. Documento de consenso de GeSIDA/Plan Nacional sobre el Sida respecto al tratamiento antirretroviral en adultos con infección por el virus de la inmunodeficiencia humana (Actualización enero 2015). Enferm Infecc Microbiol Clin 2015; 33 (8): 543.e1-43. http://dx.doi. org/10.1016/j.eimc.2015.03.016.

26.- Woodward C L, Hall A M, Williams I G, Madge S, Copas A, Nair D, et al. Tenofovirassociated renal and bone toxicity. HIV Med 2009; 10 (8): 482-7. DOI: 10.1111/j.14681293.2009.00716.x.

27.- Cooper R D, Wiebe N, Smith N, Keiser P, Naicker S, Tonelli M. Systematic review and meta-analysis: renal safety of tenofovir disoproxil fumarate in HIV-infected patients. Clin Infect Dis 2010; 51(5): 496-505. DOI: $10.1086 / 655681$

28.- Madeddu G, Bonfanti P, De Socio G V, Carradori S, Grosso C, Marconi P, et al. CISAI Group. Tenofovir renal safety in HIV-infected patients: Results from the SCOLTA Project. Biomed Pharmacother 2008; 62 (1): 6-11. DOI: 10.1016/j. biopha.2007.04.008.

29.- Jiang H Y, Zhang M N, Chen H J, Yang Y, Deng M, Ruan B. Nevirapine versus efavirenz for patients co-infected with HIV and tuberculosis: a systematic review and metaanalysis. Int J Infect Dis 2014; 25: 130-5. DOI: 10.1016/j.ijid.2014.04.020.

30.- Styrt B A, Piazza-Hepp T D, Chikami G K. Clinical toxicity of antiretroviral nucleoside analogs. Antiviral Res.1996; 31 (3): 121-35. DOI: 10.1016/0166-3542(96)85209-0.

31.- Swartz J E, Vandekerckhove L, Ammerlaan H, de Vries A C, Begovac J, Bierman W F, et al. Efficacy of tenofovir and efavirenz in combination with lamivudine or emtricitabine in antiretroviral-naive patients in Europe. $\mathrm{J}$ Antimicrob Chemother 2015; 70 (6): 1850-7. DOI: $10.1093 / \mathrm{jac} / \mathrm{dkv} 033$.

32.- Ford N, Vitoria M, Doherty M, Gray A. Candidates for inclusion in a universal antiretroviral regimen: are lamivudine and emtricitabine interchangeable? Curr Opin HIV AIDS 2017; 12 (4): 334-8. DOI: 10.1097/ COH.0000000000000377. 